\title{
ON THE ANALYTICITY OF SOLUTIONS OF FIRST-ORDER NONLINEAR PDE
}

\author{
NICHOLAS HANGES AND FRANCOIS TREVES
}

\begin{abstract}
Let $(x, t) \in R^{m} \times R$ and $u \in C^{2}\left(R^{m} \times R\right)$. We discuss local and microlocal analyticity for solutions $u$ to the nonlinear equation
\end{abstract}

$$
u_{t}=f\left(x, t, u, u_{x}\right) \text {. }
$$

Here $f\left(x, t, \zeta_{0}, \zeta\right)$ is complex valued and analytic in all arguments. We also assume $f$ to be holomorphic in $\left(\zeta_{0}, \zeta\right) \in C \times C^{m}$. In particular we show that

$$
\mathrm{WF}_{A} u \subset \operatorname{Char}\left(L^{u}\right)
$$

where $\mathrm{WF}_{A}$ denotes the analytic wave-front set and $\operatorname{Char}\left(L^{u}\right)$ is the characteristic set of the linearized operator

$$
L^{u}=\partial / \partial t-\sum \partial f / \partial \zeta_{j}\left(x, t, u, u_{x}\right) \partial / \partial x_{j} .
$$

If we assume $u \in C^{3}\left(R^{m} \times R\right)$ then we show that the analyticity of $u$ propagates along the elliptic submanifolds of $L^{u}$.

\section{INTRODUCTION}

The present article concerns $\mathscr{C}^{2}$ solutions of a nonlinear PDE

$$
u_{t}=f\left(x, t, u, u_{x}\right)
$$

where $f\left(x, t, \zeta_{0}, \zeta\right)$ is complex-valued and real analytic, and holomorphic with respect to $\left(\zeta_{0}, \zeta\right)$. Here $x$ varies in an open subset of $\mathbb{R}^{m}, t$ in an interval, and $\left(\zeta_{0}, \zeta\right)$ in an open subset of $\mathbb{C}^{m+1}$. In [Chemin, 1988] it is proved, under the weaker hypothesis that $f$ is merely $\mathscr{C}^{\infty}$ with respect to $(x, t)$, that the $\mathscr{C}^{\infty}$ wave-front set of any $\mathscr{C}^{2}$ solution $u$ is contained in the characteristic set of the linearized operator

$$
L^{u}=\partial / \partial t-\sum_{j=1}^{m}\left(\partial f / \partial \zeta_{j}\right)\left(x, t, u, u_{x}\right) \partial / \partial x_{j} .
$$

We prove the analogous theorem with $\mathscr{C}^{\infty}$ replaced by $\mathscr{C}^{\omega}$. Perhaps the result is known, though we have been unable to find it in the literature. Possibly it can be established by the methods of paradifferential calculus. Nevertheless we thought that a simple proof was worth publishing. Our proof relies solely

Received by the editors March 2, 1990.

1980 Mathematics Subject Classification (1985 Revision). Primary 35F20; Secondary 35S30, 35A20, 35A27.

Key words and phrases. Characteristic set, analytic wave-front set, Hamiltonian.

The work of F. Treves was partly supported by NSF GRANT DMS-8903007. 
on the implicit function theorem in conjunction with known results of linear theory-but the linear theory of hypo-analytic structures (see [Baouendi-ChangTreves, 1983]). Two lemmas (1.1 and 1.2) are needed, which might qualify as slightly harder analysis, on estimating the FBI transform of a solution to a linear equation. But the authors have included their proofs only to persuade the reader that what is true, and known, for linear partial differential equations with $\mathscr{C} \infty$ coefficients is also true when the coefficients are $\mathscr{C}^{1}$. Section 3 shows that the analyticity of $\mathscr{C}^{3}$ solutions $u$ of $(*)$ propagates along the elliptic submanifolds of the linearized vector field $(* *)$. This is the same phenomenon as in the linear case (see [Hanges-Treves, 1983]).

\section{Preliminaries about a first-ORder linear PDE}

We consider a complex vector field in an open neighborhood of the origin, $\Omega$, in $\mathbb{R}^{m+1}$,

$$
L=\partial / \partial t+\sum_{j=1}^{m} c_{j}(x, t) \partial / \partial x_{j},
$$

with coefficients $c_{j} \in \mathscr{C}^{1}(\Omega)$. We shall reason under the following hypothesis:

$$
\begin{aligned}
& \text { there exist } m \mathscr{C}^{1} \text { functions } \psi_{i}(1 \leq i \leq m) \text { in } \Omega \text { such that, } \\
& \text { if we set set } Z_{i}=x_{i}+t \psi_{i}(x, t) \text {, then } L Z_{i}=0 .
\end{aligned}
$$

We write $\psi=\left(\psi_{1}, \ldots, \psi_{m}\right), Z=\left(Z_{1}, \ldots, Z_{m}\right)$. We select an open neighborhood $U \Subset \Omega$ of 0 such that the mapping $U \ni(x, t) \rightarrow(Z(x, t), t) \in \mathbb{C}^{m} \times \mathbb{R}$ is a diffeomorphism of $U$ onto a $\mathscr{C}^{1}$ submanifold $\Sigma$ of $\mathbb{C}^{m} \times \mathbb{R}$. In particular the Jacobian matrix $Z_{x}$ must be nonsingular in $U$. It is convenient to take $U=\mathscr{B} \times \mathscr{I}$ with $\mathscr{B}$ an open ball in $x$-space $\mathbb{R}^{m}$ and $\mathscr{I}$ an open interval in the $t$-line $\mathbb{R}$, both centered at the origin.

We have

$$
\sum_{j=1}^{m} c_{j} \partial Z_{i} / \partial x_{j}=-\partial Z_{i} / \partial t, \quad i=1, \ldots, m
$$

whence, in vector-matrix notation,

$$
c=-Z_{x}^{-1} Z_{t} .
$$

Of course, $Z_{x}=I+t \psi_{x}, Z_{t}=\psi+t \psi_{t} \quad(I: m \times m$ identity matrix $)$. The symbol of $L$ is given by

$$
\lambda=\tau-Z_{t} \cdot{ }^{t} Z_{x}^{-1} \xi
$$

(left superscript ${ }^{t}$ indicates transposition).

We consider now the submanifold $\mathscr{X}$ of $U$ defined by $t=0$, i.e., $\mathscr{X}=$ $\mathscr{B} \times\{0\}$. On $\mathscr{X}, d Z_{i}=d x_{i}+\psi_{i}(x, 0) d t$. The characteristic set $T^{0}$ of $L$, i.e., the subset of $T^{*} \mathbb{R}^{m+1} \backslash 0 \cong \mathbb{R}^{m+1} \times\left(\mathbb{R}^{m+1} \backslash\{0\}\right)$ on which the function $\lambda$ vanishes, consists of the points $(x, t, \xi, \tau)$ such that, for some $\zeta \in \mathbb{C}^{m}$,

$$
\xi \cdot d x+\tau d t=\zeta \cdot d Z
$$

(the center dot - stands for the "real" scalar product). Over $\mathscr{X}$ this means

$$
\zeta=\xi, \tau=\operatorname{Re} \psi(x, 0) \cdot \xi, \quad \operatorname{Im} \psi(x, 0) \cdot \xi=0 .
$$


The pull-back map $\left.\mathbb{C} T^{*} \mathbb{R}^{m+1}\right|_{\mathscr{X}} \rightarrow \mathbb{C} T^{*} \mathscr{X}$ transforms $\left.T^{0}\right|_{\mathscr{X}}$ into the set

$$
((x, 0), \xi \cdot d x) \in T^{*} \mathscr{X} ; \quad \operatorname{Im} \psi(x, 0) \cdot \xi=0 .
$$

This said, consider an arbitrary $\mathscr{C}^{1}$ solution $h$ of the equation $L h=0$ in $\Omega$ and its FBI transform

$$
\mathscr{F} h(t ; z, \zeta)=\int_{\mathscr{B}} e^{\iota \zeta \cdot\left(z-Z\left(x^{\prime}, t\right)\right)-\langle\zeta\rangle\left\langle z-Z\left(x^{\prime}, t\right)\right\rangle^{2}} h\left(x^{\prime}, t\right) d Z\left(x^{\prime}, t\right) .
$$

Here $\langle\zeta\rangle=(\zeta \cdot \zeta)^{1 / 2}$ (main branch of the square-root). Notice that

$$
\mathscr{F} h(0 ; z, \zeta)=\int_{\mathscr{B}} e^{\iota \zeta \cdot\left(z-x^{\prime}\right)-\langle\zeta\rangle\left\langle z-x^{\prime}\right\rangle^{2}} h\left(x^{\prime}, 0\right) d x^{\prime} .
$$

Lemma 1.1. Let $r>0$ be the radius of the ball $\mathscr{B}$. There is an open neighborhood $\mathscr{O}$ of 0 in $\mathbb{C}^{m}$, a conic open neighborhood $\mathscr{C}$ of $\mathbb{R}^{m} \backslash\{0\}$ in $\mathbb{C}^{m} \backslash\{0\}$ and positive constants $\delta, C$ such that

$$
|\mathscr{F} h(t ; z, \zeta)-\mathscr{F} h(0 ; z, \zeta)| \leq C|t| e^{-r^{2}|\zeta| / 2} \underset{\partial \mathscr{B} \times[-\delta, \delta]}{\operatorname{Max}}|h|
$$

for all solutions $h \in \mathscr{C}^{1}(\Omega)$ of $L h=0$ and all

$$
t \in \mathbb{R}, \quad|t|<\delta, \quad z \in \mathscr{O}, \quad \zeta \in \mathscr{C} \text {. }
$$

Proof. We have

$$
\begin{aligned}
-(\partial / \partial t) & \mathscr{F} h(t ; z, \zeta) \\
= & \int_{\mathscr{B}} t_{L}\left(e^{l \zeta \cdot\left(z-Z\left(x^{\prime}, t\right)\right)-\langle\zeta\rangle\left\langle z-Z\left(x^{\prime}, t\right)\right\rangle^{2}} h\left(x^{\prime}, t\right) \operatorname{det} Z_{x}\left(x^{\prime}, t\right)\right) d x^{\prime} \\
& +\int_{\mathscr{B}} \sum_{j=1}^{m}\left(\partial / \partial x_{j}\right)\left(c_{j} e^{l \zeta \cdot\left(z-Z\left(x^{\prime}, t\right)\right)-\langle\zeta\rangle\left\langle z-Z\left(x^{\prime}, t\right)\right\rangle^{2}} h\left(x^{\prime}, t\right) \operatorname{det} Z_{x}\left(x^{\prime}, t\right)\right) d x^{\prime} .
\end{aligned}
$$

The first integral is equal to zero due to the hypothesis that $L h=0$. Integration in the second integral shows that

$$
\begin{aligned}
&-(\partial / \partial t) \mathscr{F} h(t ; z, \zeta) \\
& \quad=\int_{\partial \mathscr{B}} e^{i \zeta \cdot\left(z-Z\left(x^{\prime}, t\right)\right)-\langle\zeta\rangle\left\langle z-Z\left(x^{\prime}, t\right)\right\rangle^{2}} h\left(x^{\prime}, t\right) a\left(x^{\prime}, t\right) d \sigma\left(v^{\prime}, .\right.
\end{aligned}
$$

Putting $z=0, \zeta \in \xi \in \mathbb{R}^{m} \backslash\{0\}$ shows that

$$
\begin{aligned}
& -(\partial / \partial t) \mathscr{F} h(t ; z, \zeta) \\
& \quad=\int_{\partial \mathscr{B}} e^{-l \xi \cdot\left(x^{\prime}+t \psi\left(x^{\prime}, t\right)\right)-|\xi|\left\langle x^{\prime}+t \psi\left(x^{\prime}, t\right)\right\rangle^{2}} h\left(x^{\prime}, t\right) a\left(x^{\prime}, t\right) d \sigma\left(x^{\prime}\right)
\end{aligned}
$$

for a suitable choice of the density $a(d \sigma$ is the spherical measure on $\partial \mathscr{B})$. In the integral over $\partial \mathscr{B}$ in $(1.8)$ we have

$$
\begin{aligned}
\operatorname{Im}\{-\xi & \left.\cdot\left[x^{\prime}+t \psi\left(x^{\prime}, t\right)\right]+t|\xi|\left\langle x^{\prime}+t \psi\left(x^{\prime}, t\right)\right\rangle^{2}\right\} /|\xi| \\
= & t \operatorname{Im} \psi\left(x^{\prime}, t\right) \cdot \dot{\xi}+r^{2}+2 t \operatorname{Re} \psi\left(x^{\prime}, t\right) \cdot x^{\prime} \\
+ & t^{2}\left|\operatorname{Re} \psi\left(x^{\prime}, t\right)\right|^{2}-t^{2}\left|\operatorname{Im} \psi\left(x^{\prime}, t\right)\right|^{2}
\end{aligned}
$$

where $\dot{\xi}=\xi /|\xi|$. We select $\delta>0$ small enough that

$$
\left|t \operatorname{Im} \psi\left(x^{\prime}, t\right) \cdot \dot{\xi}\right|+2\left|t x^{\prime} \cdot \operatorname{Re} \psi\left(x^{\prime}, t\right)\right|+t^{2}\left|\operatorname{Im} \psi\left(x^{\prime}, t\right)\right|^{2}<r^{2} / 4
$$


for all $x^{\prime} \in \partial \mathscr{B},|t| \leq \delta$. From (1.8) and (1.9) we derive

$$
|(\partial / \partial t) \mathscr{F} h(t ; 0, \xi)| \leq C e^{-3 r^{2}|\xi| / 4} \underset{\partial \mathscr{B} \times[-\delta, \delta]}{\operatorname{Max}}|h| .
$$

If we allow $z$ to vary in $\mathscr{O}$ and $\zeta$ to vary in the cone $\mathscr{C}$, provided $\Omega$ and the conical distance from $\mathbb{C}^{m} \backslash \mathscr{C}$ to $\mathbb{R}^{m}$ are sufficiently small, we obtain

$$
|(\partial / \partial t) \mathscr{F} h(t ; z, \zeta)| \leq C e^{-r^{2}|\zeta| / 2} \underset{\partial \mathscr{B} \times[-\delta, \delta]}{\operatorname{Max}}|h| .
$$

Integration from 0 to $t$ shows that (1.7) is a consequence of (1.10).

We shall now restrict the variation of $\zeta$ to a conic neighborhood $\mathscr{C}^{0}$ in $\mathbb{C}^{m} \backslash\{0\}$ of a point $\xi^{0} \in S^{m-1}$ (the unit sphere in $\mathbb{R}^{m}$ ) such that no characteristic point of $L$ lies above $\left(0, \xi^{0}\right) \in T^{*} \mathscr{X}$. By what was said above this means that $\operatorname{Im} \psi(0,0) \cdot \xi^{0} \neq 0$. Possibly after replacing $\xi^{0}$ by $-\xi^{0}$ we may always assume that

$$
\operatorname{Im} \psi(0,0) \cdot \xi^{0}>0 .
$$

Lemma 1.2. Suppose (1.11) holds. Then there is an open neighborhood of 0 in $\mathbb{C}^{m}, \mathscr{O}$, an open conic neighborhood $\mathscr{C}^{0}$ of $\xi^{0} \in S^{m-1}$ in $\mathbb{C}^{m}$ and constants $C, \delta_{1}>0$ such that

$$
|\mathscr{F} h(t ; z, \zeta)| \leq C e^{t\left(\operatorname{Im} \psi(0,0) \cdot \xi^{0}\right)|\zeta| / 2} \underset{\mathscr{B} \times[-\delta, \delta]}{\operatorname{Max}}|h|
$$

for all solutions $h \in \mathscr{C}^{1}(\Omega)$ of $L h=0$ and all

$$
t \in \mathbb{R}, \quad-\delta_{1}<t<0, \quad z \in \mathscr{O}, \quad \zeta \in \mathscr{C}^{0} .
$$

Proof. We have, for $t \in \mathbb{R}$ and $\rho>0$,

$$
\mathscr{F} h\left(t ; 0, \rho \xi^{0}\right)=\int_{\mathscr{B}} e^{-\imath \rho \xi^{0} \cdot\left(x^{\prime}+t \psi\left(x^{\prime}, t\right)\right)-\rho\left\langle x^{\prime}+t \psi\left(x^{\prime}, t\right)\right\rangle^{2}} h\left(x^{\prime}, t\right) d Z\left(x^{\prime}, t\right) .
$$

The hypothesis that $\psi$ is of class $\mathscr{C}^{1}$ allows us to write, for some constant $K>0$ and all $x^{\prime} \in \mathscr{B}, t \in \mathscr{J}, t \leq 0$,

$$
\begin{aligned}
\operatorname{Im}\{- & \left.\xi^{0} \cdot\left[x^{\prime}+t \psi\left(x^{\prime}, t\right)\right]+l\left\langle x^{\prime}+t \psi\left(x^{\prime}, t\right)\right\rangle^{2}\right\} \\
= & -t \operatorname{Im} \psi\left(x^{\prime}, t\right) \cdot \xi^{0}+\left|x^{\prime}\right|^{2}+2 t \operatorname{Re} \psi\left(x^{\prime}, t\right) \cdot x^{\prime} \\
& \quad+t^{2}\left|\operatorname{Re} \psi\left(x^{\prime}, t\right)\right|^{2}-t^{2}\left|\operatorname{Im} \psi\left(x^{\prime}, t\right)\right|^{2} \\
\geq & |t| \operatorname{Im} \psi(0,0) \cdot \xi^{0}+O\left[|t|\left(\left|x^{\prime}\right|+|t|\right)\right]+\left|x^{\prime}\right|^{2} \\
\geq & -t\left(\operatorname{Im} \psi(0,0) \cdot \xi^{0}\right)-K t^{2} .
\end{aligned}
$$

If we require

$$
t<0, \quad|t| \leq \frac{1}{4} K^{-1} \operatorname{Im} \psi(0,0) \cdot \xi^{0}=\delta_{1}
$$

and select $\mathscr{O}$ small enough and $\mathscr{C}^{0}$ sufficiently "thin" about the ray through $\xi^{0}$ we reach the desired conclusion.

If we combine Lemmas 1.1 and 1.2 we get 
Lemma 1.3. Suppose (1.11) holds. Then there is an open neighborhood of 0 in $\mathbb{C}^{m}, \mathscr{O}$, an open conic neighborhood $\mathscr{C}^{0}$ of $\xi^{0} \in S^{m-1}$ in $\mathbb{C}^{m}$ and constants $C, c_{0}>0$, such that

$$
|\mathscr{F} h(0 ; z, \zeta)| \leq C e^{-c_{0}|\zeta|} \operatorname{Max}_{\mathscr{B} \times[-\delta, \delta]}|h|
$$

for all solutions $h \in \mathscr{C}^{1}(\Omega)$ of $L h=0$ and all $z \in \mathscr{O}, \zeta \in \mathscr{C}^{0}$.

Let us now reason in $(x, t, \xi, \tau)$-space. We look at the characteristic points that lie above the origin $(x=0, t=0)$. As pointed out earlier they are given by

$$
\tau-\operatorname{Re} \psi(0,0) \cdot \xi=0, \quad \operatorname{Im} \psi(0,0) \cdot \xi=0 .
$$

It follows that a point $\left(0,0, \xi^{0}, \tau^{0}\right) \in \mathbb{R}^{m+1} \times \mathbb{R}^{m+1}$ is noncharacteristic if any one of the two equations (1.15) is violated.

We introduce an extra variable $s \in \mathbb{R}$, a parameter $\theta \in[0,2 \pi)$ and we define the vector field in $\mathbb{R} \times \Omega$,

$$
\mathscr{L}^{\theta}=\partial / \partial s-e^{-\imath \theta} L .
$$

We note that every solution $h \in \mathscr{C}^{1}(\Omega)$ of $L h=0$ can be regarded as a solution of the equation $\mathscr{L}^{\theta} h=0$ in $\mathbb{R} \times \Omega$.

Let $\mathscr{I}_{0}$ be an open interval in $\mathbb{R}$ centered at the origin. We shall assume that $\mathscr{L}^{\theta}$ satisfies hypothesis $(1.1)$ in the open set $\mathscr{I}_{0} \times \Omega$. This means that there are $m$ functions $\psi_{i}^{\theta} \in \mathscr{C}^{1}\left(\mathscr{J}_{0} \times \Omega\right)(1 \leq i \leq m)$ such that, if $Z_{i}^{\theta}=$ $x_{i}+s \psi_{i}^{\theta}(x, s, t)$, then $\mathscr{L}^{\theta} Z_{i}^{\theta}=0$. Actually we need one more "first integral," one whose value at $s=0$ is equal to $t$; it suffices to take $Z_{m+1}^{\theta}=t+e^{-i \theta} s$.

We observe that, above the points $x=0, t=0$,

$$
\operatorname{symb}\left(\mathscr{L}^{\theta}\right)=\sigma-e^{-i \theta}\left(\tau+\sum_{j=1}^{m} c_{j}(0,0) \xi_{j}\right) .
$$

On the other hand, by (1.2) we also have

$$
c_{j}(0,0)=-\psi_{j}(0,0), \quad j=1, \ldots, m
$$

[here $\psi_{j}$ is the same function as in (1.1)]. It follows that, when $x=0, t=0$

$$
\begin{aligned}
\operatorname{symb}\left(\mathscr{L}^{\theta}\right)= & \sigma-[(\tau-\operatorname{Re} \psi \cdot \xi) \cos \theta-(\operatorname{Im} \psi \cdot \xi) \sin \theta] \\
& +l[(\tau-\operatorname{Re} \psi \cdot \xi) \sin \theta+(\operatorname{Im} \psi \cdot \xi) \cos \theta] .
\end{aligned}
$$

We introduce the FBI transform with respect to $(x, t)$ :

$$
\widehat{\mathscr{F}} h(z, w, \zeta, \tau)=\int_{U} e^{l\left[\zeta \cdot\left(z-x^{\prime}\right)+\tau\left(w-t^{\prime}\right)\right]-\langle(\zeta, \tau)\rangle\left[\left\langle z-x^{\prime}\right\rangle^{2}+\left(w-t^{\prime}\right)^{2}\right]} h\left(x^{\prime}, t^{\prime}\right) d x^{\prime} d t^{\prime} .
$$

This is the same as the FBI transform defined above, but in $(x, s, t)$-space, and computed on the submanifold $s=0$. If we apply Lemma 1.3 we reach the following conclusion:

Lemma 1.4. Suppose that, for some $\theta \in[0,2 \pi)$,

$$
[\tau-\operatorname{Re} \psi(0,0) \cdot \xi] \sin \theta+[\operatorname{Im} \psi(0,0) \cdot \xi] \cos \theta \neq 0 \text {. }
$$


Then there is an open neighborhood of 0 in $\mathbb{C}^{m}, \mathscr{O}$, an open conic neighborhood $\mathscr{C}^{0}$ of $\xi^{0} \in S^{m-1}$ in $\mathbb{C}^{m}$ and constants $C, c_{0}>0$ such that

$$
|\widehat{\mathscr{F}} h(z, w, \zeta, \tau)| \leq C e^{-c_{0}|\zeta|} \operatorname{Max}_{U}|h|
$$

for all solutions $h \in \mathscr{C}^{1}(\Omega)$ of $L h=0$ and all $z \in \mathscr{O}, \zeta \in \mathscr{C}^{0}$.

It is clear that the set of points $(\xi, \tau)$ such that there is $\theta \in[0,2 \pi)$ such that $(1.18)$ holds is the same as the set of points $(\xi, \tau)$ which violate $(1.15)$. Thanks to the characterization of the analytic wave front set of a distribution $u, W F_{a} u$, by the exponential decay of its FBI transform we may state

Theorem 1.1. Suppose that, for some choice of the interval $\mathscr{I}_{0} \subset \mathbb{R}$ centered at the origin and for every $\theta \in[0,2 \pi)$, the vector field $\mathscr{L}^{\theta}$ satisfies condition (1.1) in $\mathscr{I}_{0} \times \Omega$. Then, at the origin, the analytic wave-front set of every solution $h \in \mathscr{C}^{1}(\Omega)$ of the equation $L h=0$ is contained in the characteristic set of $L$.

Remark 1.1. Suppose the vector field $L$ is elliptic, which requires $m=1$, and that $L$ satisfies (1.1). Then, in some open neighborhood of the origin, an arbitrary solution $h$ of the equation $L h=0$ has the form $\tilde{h}(Z)$ with $\tilde{h}$ a holomorphic function in some open neighborhood of the origin in the complex plane. As a consequence of this and of $(1.1) h(x, 0)=\tilde{h}(x)$ which shows that $h(x, 0)$ is analytic at the origin. This is in agreement with the conclusion in Lemma 1.3.

Remark 1.2. Assume that the coefficients of $L$ are analytic. Then condition (1.1) is always satisfied. We derive from Lemma 1.3 that the analytic wavefront set, at $x=0$, of each trace of an arbitrary solution $h$ of $L h=0$ on a hyperplane $t=$ const. is contained in the pull-back to that hyperplane of the characteristic set of $L$. This result implies that the analytic wave-front set of $h$ itself is contained in the characteristic set of $L$, by the argument used to prove Lemma 1.4 , since clearly $\mathscr{L}^{\theta}$ also satisfies (1.1). This complements the arguments on the hypo-analytic wave-front set of solutions in [Baouendi-ChangTreves, 1983].

Finally we shall need the following result.

Lemma 1.5. Suppose there is an open interval $\mathscr{I}_{0}$ such that the following is true. For every $\theta \in[0,2 \pi)$ the vector field $\mathscr{L}^{\theta}$ satisfies condition (1.1) in $\mathscr{I}_{0} \times \Omega$; $h(x, t, \lambda) \in \mathscr{C}^{1}(\Omega)$ is a solution of $L h=0$ which depends analytically on $\lambda \in \mathscr{I}$. Then the analytic wave-front set of $h(x, t, t)$ at the origin is contained in $T_{0}^{0}$.

Proof. We extend $h(x, t, \lambda)$ as a holomorphic function of $\lambda$ in an open disk $\Delta \subset \mathbb{C}^{1}$ centered at 0 , valued in $\mathscr{C}^{1}(U)$ (that such a disk exists is what we mean by saying that $h$ depends analytically on $\lambda)$. Consider a unit covector $\left(\xi^{0}, \tau^{0}\right) \notin T_{0}^{0}$; there is a conic open neighborhood of $\left((0,0),\left(\xi^{0}, \tau^{0}\right)\right)$ in which the FBI transform of $h$ decays exponentially (as $|\xi|+|\tau| \rightarrow+\infty$; see Lemma 1.4). Inspection of the inversion formula for the FBI transform shows that there are finitely many open convex cones in $\mathbb{R}^{m+1} \backslash\{0\}, \Gamma_{1}, \ldots, \Gamma_{r}$, independent of $\lambda \in \Delta$ (possibly contracted about 0 ) and such that

$$
\xi^{0} \cdot y+\tau^{0} s<0, \quad \forall(y, s) \in \Gamma_{j}(1 \leq j \leq r),
$$


and an equal number of distributions $h_{1}, \ldots, h_{r}$ depending holomorphically on $\lambda$ in $\Delta$, such that, in some open neighborhood $V \subset U$ of the origin in $\mathbb{R}^{m+1}$

$$
h=h_{1}+\cdots+h_{r},
$$

and that, for each $j=1, \ldots, r, h_{j}$ is the distribution boundary value, as $(z, w) \rightarrow(x, t)$, of a holomorphic function $\tilde{h}_{j}(z, w, \lambda)$ in $\left(V+\imath \Gamma_{j}\right) \times \Delta$. We observe that $h_{j}(x, t, t)$ is the boundary value (in $\left.V\right)$ of $\tilde{h}_{j}(z, w, w)$ which is obviously a holomorphic function in the wedge

$$
\mathscr{W}_{j}=\left\{(z, w) \in V+\imath \Gamma_{j} ; w \in \Delta\right\},
$$

whence the claim.

\section{Application to First-ORDER NONLINEAR PDE}

Same notation as in $\S 1$. In what follows $f\left(z, w, \zeta_{0}, \zeta\right)$ will denote a holomorphic function in an open neighborhood $\widetilde{\Omega} \times \mathcal{N}$ of $((0,0),(a, \omega))$ in $\mathbb{C}^{m+1} \times \mathbb{C}^{m+1}$. We assume $U \subset \Omega \subset \widetilde{\Omega} \cap \mathbb{R}^{m+1}$ and study a solution $u \in \mathscr{C}^{2}(U)$ of the nonlinear differential equation

$$
u_{t}=f\left(x, t, u, u_{x}\right)
$$

under the hypothesis that

$$
u(0,0)=a, \quad u_{x}(0,0)=\omega ; \quad \forall(x, t) \in U,\left(u(x, t), u_{x}(x, t)\right) \in \mathcal{N} .
$$

We introduce the vector field in $\Omega$, depending on the parameters $\left(\zeta_{0}, \zeta\right) \in \mathscr{N}$,

$$
\mathscr{L}=\partial / \partial t-\sum_{j=1}^{m}\left(\partial f / \partial \zeta_{j}\right)\left(x, t, \zeta_{0}, \zeta\right) \partial / \partial x_{j} .
$$

We form

$$
L^{u}=\partial / \partial t-\sum_{j=1}^{m}\left(\partial f / \partial \zeta_{j}\right)\left(x, t, u, u_{x}\right) \partial / \partial x_{j}
$$

$L^{u}$ is a vector field with $\mathscr{C}^{1}$ coefficients in $U$.

We differentiate both sides of (2.1) with respect to $x$ and we introduce a vector-valued function $\mathbf{v}=\left(u, u_{x_{1}}, \ldots, u_{x_{m}}\right) \in \mathscr{C}^{1}\left(\Omega ; \mathbb{C}^{m+1}\right)$; by $(2.2) \mathbf{v}(U) \subset$ $\mathcal{N}$. We may rewrite equation $(2.1)$ as

$$
L^{u} u=g_{0}(x, t, \mathbf{v})
$$

where

$$
g_{0}=f-\sum_{j=1}^{m} \zeta_{j} \partial f / \partial \zeta_{j} .
$$

For $i=1, \ldots, m$ we have

$$
L^{u} u_{x_{i}}=g_{i}(x, t, \mathbf{v}),
$$

where

$$
g_{i}=f_{x_{i}}+\zeta_{i}\left(\partial f / \partial \zeta_{0}\right)
$$


We can regard the system of equations $(2.3)_{0},(2.3)_{i} \quad(1 \leq i \leq m)$, as a quasilinear system of differential equations

$$
L^{u} \mathbf{v}=\mathbf{g}(x, t, \mathbf{v}) .
$$

Here $\mathbf{g}\left(x, t, \zeta_{0}, \zeta\right)$ extends as a holomorphic function in $\widetilde{\Omega} \times \mathscr{N}$; and $\mathbf{v}$ is a $\mathscr{C}^{1}$ map of $U$ into $\mathcal{N}$.

We introduce the principal part of the (holomorphic) Hamiltonian of the system (2.4):

$$
H=\mathscr{L}+g_{0} \partial / \partial \zeta_{0}+\sum_{j=1}^{m} g_{j} \partial / \partial \zeta_{j}
$$

Now let $\mathbf{h}$ be any $\mathscr{C}^{1}$ map $U \rightarrow \mathscr{N}$ such that $\mathbf{h}(0)=(a, \omega)$; if $\Psi\left(x, t, \zeta_{0}, \zeta\right)$ is any holomorphic function in $\widetilde{\Omega} \times \mathscr{N}$ we set $\Psi^{\mathbf{h}}(x, t)=\Psi(x, t, \mathbf{h}(x, t))$. We denote by $\mathscr{L}^{\mathbf{h}}$ the vector field in $U$ obtained by substituting $\mathbf{h}(x, t)$ for $\left(\zeta_{0}, \zeta\right)$ in each coefficient of $\mathscr{L}$. [In this notation $\mathscr{L}^{\mathbf{v}}=L^{u}$.] Direct computation shows that

$$
\begin{aligned}
\mathscr{L}^{\mathbf{h}} \Psi^{\mathbf{h}}= & (H \Psi)^{\mathbf{h}}+\left(\partial \Psi / \partial \zeta_{0}\right)^{\mathbf{h}}\left(\mathscr{L}^{\mathbf{h}} h_{0}-g_{0}^{\mathbf{h}}\right) \\
& +\sum_{j=1}^{m}\left(\partial \Psi / \partial \zeta_{j}\right)^{\mathbf{h}}\left(\mathscr{L}^{\mathbf{h}} h_{j}-g_{j}^{\mathbf{h}}\right) .
\end{aligned}
$$

We solve the following Cauchy problems

$$
\begin{array}{ll}
H Z_{i}=0, & \left.Z_{i}\right|_{t=0}=x_{i}(1 \leq i \leq m) ; \\
H \Xi_{j}=0, & \left.\Xi_{j}\right|_{t=0}=\zeta_{j}(0 \leq j \leq m) .
\end{array}
$$

Possibly after contracting $\widetilde{\Omega}$ about 0 and $\mathscr{N}$ about $(a, \omega)$ there are unique holomorphic solutions $Z_{i}$ and $\Xi_{j}$ in $\widetilde{\Omega} \times \mathscr{N}$. We substitute them for $\Psi$ in (2.5). We obtain

$$
\begin{array}{r}
\mathscr{L}^{\mathbf{h}} Z_{i}^{\mathbf{h}}=\left(\partial Z_{i} / \partial \zeta_{0}\right)^{\mathbf{h}}\left(\mathscr{L}^{\mathbf{h}} h_{0}-g_{0}^{\mathbf{h}}\right)+\sum_{j=1}^{m}\left(\partial Z_{i} / \partial \zeta_{j}\right)^{\mathbf{h}}\left(\mathscr{L}^{\mathbf{h}} h_{j}-g_{j}^{\mathbf{h}}\right), \\
i=1, \ldots, m ; \\
\mathscr{L}^{\mathbf{h}} \Xi_{i}^{\mathbf{h}}=\left(\partial \Xi_{i} / \partial \zeta_{0}\right)^{\mathbf{h}}\left(\mathscr{L}^{\mathbf{h}} h_{0}-g_{0}^{\mathbf{h}}\right)+\sum_{j=1}^{m}\left(\partial \Xi_{i} / \partial \zeta_{j}\right)^{\mathbf{h}}\left(\mathscr{L}^{\mathbf{h}} h_{j}-g_{j}^{\mathbf{h}}\right), \\
i=0,1, \ldots, m .
\end{array}
$$

We shall write $Z=\left(Z_{1}, \ldots, Z_{m}\right), \Xi=\left(\Xi_{0}, \Xi_{1}, \ldots, \Xi_{m}\right)$. Since the Jacobian determinant $\left(\partial \Xi_{i} / \partial \zeta_{j}\right)_{0 \leq i, j<1}$ is nonzero in a full neighborhood of $(0,0, a, \omega)$ we see that, the validity of $(2.4)$ in some neighborhood of 0 is equivalent to the validity, in such a neighborhood, of the system of differential equations

$$
\mathscr{L}^{\mathrm{v}} \Xi_{i}^{\mathrm{v}}=0, \quad i=0,1, \ldots, m .
$$

And (2.4) entails

$$
\mathscr{L}^{\mathbf{v}} Z_{i}^{\mathrm{v}}=0, \quad i=1, \ldots, m .
$$


Note that

$$
\left.Z^{\mathbf{v}}\right|_{t=0}=x,\left.\quad \Xi^{\mathbf{v}}\right|_{t=0}=\mathbf{v}(x, 0) .
$$

It is clear that we may apply the implicit function theorem (in the holomorphic category) and solve the equations

$$
Z\left(x, t, \zeta_{0}, \zeta\right)=Z, \quad \Xi\left(x, t, \zeta_{0}, \zeta\right)=\Xi
$$

with respect to $\left(x, \zeta_{0}, \zeta\right)$ :

$$
x=P(Z, t, \Xi), \quad\left(\zeta_{0}, \zeta\right)=Q(Z, t, \Xi),
$$

with $P\left(0,0, \zeta_{0}, \zeta\right)=0, Q(0,0, a, \omega)=(a, \omega)$. We derive

$$
\mathbf{v}=Q\left(Z^{\mathbf{v}}(x, t), t, \Xi^{\mathbf{v}}(x, t)\right) \text {. }
$$

The vector field (in $\mathbb{R} \times U$, with $0 \leq \theta \leq 2 \pi$ fixed)

$$
\partial / \partial s-e^{-\imath \theta} \mathscr{L}^{\mathbf{v}}=\left(\partial / \partial s-e^{-l \theta} \mathscr{L}\right)^{\mathbf{v}}
$$

has first integrals as required in condition (1.1) (with $s$ replacing $t$ ). This simply follows from the fact that $(2.15)$ originates with the nonlinear equation $u_{s}=e^{-i \theta}\left(u_{t}-f\left(x, t, u, u_{x}\right)\right)$ which is of the same kind as $(2.1)$. If $\mathscr{J}_{0}$ is a sufficiently small open interval in the real line, centered at 0 , the function $Q\left(Z^{\mathrm{v}}(x, t), t^{\prime}, \Xi^{\mathrm{v}}(x, t)\right) \in \mathscr{C}^{1}\left(U \times \mathscr{J}_{0}\right)$ is a solution of the homogeneous equation $\mathscr{L}^{\mathbf{v}} \mathbf{h}=0$ and is analytic with respect to $t^{\prime} \in \mathscr{J}_{0}$. We are in a position to apply Lemma 1.5 and, returning to the solution $u$ of $(2.1)$, to state

Theorem 2.2. Let $u \in \mathscr{C}^{2}(U)$ be a solution of the nonlinear differential equation (2.1). The analytic wave-front set of $u$ is contained in the characteristic set of the linearized differential operator $L^{u}$.

Remark 2.1. Obviously if we restrict our attention to a quasilinear equation such as (2.4),

$$
\mathbf{v}_{t}-\sum_{j=1}^{m} a_{j}(x, t, \mathbf{v}) \mathbf{v}_{x_{j}}=\mathbf{g}(x, t, \mathbf{v})
$$

the conclusion that the analytic wave-front set of $\mathbf{v}$ is contained in the characteristic set of the linearized differential operator

$$
\mathscr{L}^{\mathbf{v}}=\partial / \partial t-\sum_{j=1}^{m} a_{j}(x, t, \mathbf{v}) \partial / \partial x_{j}
$$

remains valid under the weaker hypothesis that $\mathbf{v}$ be of class $\mathscr{C}^{1}$. [As usual we are assuming that $\mathbf{v}$ and $\mathbf{g}$ are vector-valued, that the coefficients $a_{j}$ are scalar and that they, as well as the right-hand side $\mathbf{g}$, can be extended as holomorphic functions of their arguments in some open neighborhood of $(0,0, \mathbf{v}(0,0))$ in complex space.]

\section{Propagation of analyticity of solutions of FIRST-ORDER NONLINEAR PDE}

We return to the complex vector field $L$ of $\S 1$. We shall assume that hypothesis (1.1) holds in a neighborhood of every point of $\Omega$. However we shall strengthen our requirements on the regularity of the functions $\psi_{i}$ and by way 
of consequence, of the "first integrals" $Z_{i}$. We shall hypothesize that they are of class $\mathscr{C}^{2}$ in $\Omega$ :

Given any point $\left(x_{0}, t_{0}\right)$ of $\Omega$ there exist $\mathscr{C}^{2}$ functions

$\psi_{i}\left(x, t, x_{0}, t_{0}\right)(1 \leq i \leq m)$ in an open neighborhood $U_{0}$

of $\left(x_{0}, t_{0}\right)$ such that, if we set $Z_{i}\left(x, t, x_{0}, t_{0}\right)=$

$x_{i}-x_{0_{i}}+\left(t-t_{0}\right) \psi_{i}\left(x, t, x_{0}, t_{0}\right)$, then $L Z_{i}=0$ in $U_{0}$.

Moreover, we suppose there is a $\mathscr{C}^{2}$ submanifold $\Lambda$ of $\Omega$ endowed with the following property. Let $\left(x_{0}, t_{0}\right)$ be an arbitrary point of $\Lambda$ and let $Z_{i}=$ $Z_{i}\left(x, t, x_{0}, t_{0}\right)$ be the functions in (3.1) (referred to in the sequel as "first integrals"). Then

the pull-backs to $\Lambda$ of $d Z_{1}, \ldots, d Z_{m}$ span a vector subbundle $T_{\Lambda}^{\prime}$ of $\mathbb{C} T^{*} \Lambda$ such that $T_{\Lambda}^{\prime} \cap \bar{T}_{\Lambda}^{\prime}=0$.

The vector bundle $T_{\Lambda}^{\prime}$ defines an elliptic hypo-analytic structure on $\Lambda$ and we refer to $\Lambda$ as an elliptic submanifold. Near an arbitrary point $\wp \in \Lambda$ we may assume that a number of pull-backs $\pi_{\Lambda}^{*} d Z_{i}$ form a basis of $T_{\Lambda}^{\prime}$; suppose for the sake of the argument that these correspond to $i=1, \ldots, r=\operatorname{rank}$ of $T_{\Lambda}^{\prime}$. Then (3.2) demands that

$$
\pi_{\Lambda}^{*} d Z_{1} \wedge \cdots \wedge \pi_{\Lambda}^{*} d Z_{r} \wedge \pi_{\Lambda}^{*} d \bar{Z}_{1} \wedge \cdots \wedge \pi_{\Lambda}^{*} d \bar{Z}_{r} \neq 0
$$

If $h$ is any $\mathscr{C}^{1}$ function in some neighborhood of $\wp$ in $\Lambda$ whose differential is a section of $T_{\Lambda}^{\prime}$ then there is a holomorphic function $H$ in an open neighborhood of $\left(Z_{1}(\wp), \ldots, Z_{r}(\wp)\right) \in \mathbb{C}^{r}$ such that $h=H \circ\left(Z_{1}, \ldots, Z_{r}\right)$ over a full neighborhood of $\wp$ in $\Lambda$. Let $\mathscr{V}_{\Lambda}$ be the vector subbundle of $\mathbb{C} T \Lambda$ orthogonal to $T_{\Lambda}^{\prime}$; a vector field $L$ in $\Lambda$ is a section of $\mathscr{V}_{\Lambda}$ if $L\left(\left.Z_{i}\right|_{\Lambda}\right)=0$ for all $i=1, \ldots, r$. If $L_{1}, \ldots, L_{\nu}$ form a basis of $\mathscr{V}$ over some open subset of $\Lambda$ the common real zero-set of the symbols of the vector fields $L_{j}(j=1, \ldots, \nu)$ is the zero section of $T^{*} \Lambda$; which is the reason for the name "elliptic structure." (On all this see [Treves, 1991].) We are going to apply the main result in [Hanges-Treves, 1983]:

Lemma 3.1. Suppose $Z_{1}, \ldots, Z_{m} \in \mathscr{C}^{2}(\Omega)$ and the elliptic submanifold $\Lambda$ of $\Omega$ is of class $\mathscr{C}^{2}$ and connected. Let $h$ be a $\mathscr{C}^{1}$ solution of $L h=0$ in $\Omega$. Suppose that, in some neighborhood in $\Omega$ of a point $\wp \in \Lambda, h=\tilde{h} \circ Z$ with $\tilde{h}$ holomorphic in some neighborhood of $Z(\wp)$ in $\mathbb{C}^{m}$. Then the same property holds at every other point of $\Lambda$.

In [Hanges-Treves, 1983] Lemma 3.1 is proved under the hypothesis that the first integrals $Z_{i}$ and the submanifold $\Lambda$ are of class $\mathscr{C} \infty$; but inspection of the proof show that $\mathscr{C}^{2}$ suffices. See also [Trepreau, 1990].

We go now to the nonlinear equation (2.1). Here we assume that $u$ is a solution of class $\mathscr{C}^{3}$ in the whole of $\Omega$. Solving the Cauchy problem at $t=t_{0}$ for the vector field $\mathscr{L}$ of $\S 2$ shows that hypothesis $(3.1)$ is satisfied.

Theorem 3.1. If the solution $u \in \mathscr{C}^{3}(\Omega)$ of equation (2.1) is analytic in an open neighborhood of a point of an elliptic submanifold $\Lambda$ of $\Omega$ in the sense of the linearized operator $L^{u}$ it is analytic in a full neighborhood of $\Lambda$.

Proof. We go to the quasilinear system (2.4); here $\mathbf{v} \in \mathscr{C}^{2}\left(\Omega ; \mathbb{C}^{m+1}\right)$ is a solution in the whole of $\Omega$. Assume that the origin lies on $\Lambda$ and that $u$, 
and therefore $\mathbf{v}$, are analytic in an open neighborhood of 0 in $\Omega$. Let then $U=\mathscr{B} \times \mathscr{I}$ be an open neighborhood of 0 such that there exist analytic solutions $Z_{i} \quad(1 \leq i \leq m), \Xi_{j} \quad(0 \leq j \leq m)$ of $(2.6)-(2.7)$ in $U \times \mathscr{N}[\mathcal{N}$ is the open neighborhood of $(u(0), d u(0))$ in $\mathbb{C}^{m+1}$ in $\left.\S 2\right)$. By hypothesis $x \rightarrow \mathbf{v}(x, 0)$ is an analytic function in an open neighborhood $\mathscr{B}^{\prime} \subset \mathscr{B}$ of the origin. It follows that $x \rightarrow \Xi^{\mathrm{v}}(x, 0)$ is an analytic function $H(x)$ in $\mathscr{B}^{\prime}$, valued in $\mathbb{C}^{m+1}$. But then the components $H_{i}\left(Z^{\mathbf{v}}(x, t)\right) \quad(0 \leq i \leq m)$ are solutions of the homogeneous equation $\mathscr{L}^{\mathbf{v}} h=0$ (see $(2.10),(2.11)$ ). Since $\Xi^{\mathrm{v}}=H \circ Z^{\mathrm{v}}$ when $t=0$ it must also be true in a full neighborhood of the origin in $\Omega$, by the uniqueness in the Cauchy problem in locally integrable structures (a consequence of the approximation formula of [Baouendi-Treves, 1981]). By Lemma 3.1 the analogous property must hold at every point of $\Lambda \cap U$.

We apply the implicit function theorem (in the holomorphic category) with respect to $\left(\zeta_{0}, \zeta\right)$ to the equations

$$
\Xi=\Xi\left(x, t, \zeta_{0}, \zeta\right)
$$

getting $\left(\zeta_{0}, \zeta\right)=\Phi(z, t, \Xi)$. We get

$$
\mathbf{v}=\Phi\left(x, t, \Xi^{\mathbf{v}}\right) \text {. }
$$

Consider now an arbitrary point $\wp=\left(x_{0}, t_{0}\right) \in \Lambda \cap U$; in some neighborhood of $\wp$ in $\Omega$ we may write $\Xi^{\mathbf{v}}=H\left(Z^{v}\right)$. Since the Jacobian of the map $Z$ with respect to $x$ is close to 1 we see that the first partial derivatives of the functions $H_{i}$ will be bounded independently of $\wp$ (though the function $H$ may change from point to point). Using this fact and the fact that the partial derivatives of the $Z_{i}$ with respect to $\left(\zeta_{0}, \zeta\right)$ are as close to zero as we wish (provided we contract $U)$ we may apply the implicit function theorem with respect to $\left(\zeta_{0}, \zeta\right)$ to the equations

$$
\zeta_{j}=\Phi_{j}(x, t, H[Z(x, t, \zeta)]), \quad j=0,1, \ldots, m,
$$

thus getting $\zeta_{j}=G_{j}(x, t)$ with $G_{j}$ analytic in some open neighborhood of $\left(x_{0}, t_{0}\right)$. But then, by $(3.3), \mathbf{v}_{j}=G_{j}(x, t)$ in that same neighborhood. We conclude that $\mathbf{v}$, and therefore $u$, is analytic in a neighborhood of $\Lambda \cap U$. This shows easily that the subset of points of $\Lambda$ in a neighborhood of which $u$ is analytic is closed; since it is trivially open this completes the proof of Theorem 3.1.

Remark 3.1. One could likewise apply Theorem 2.2 of [Trepreau, 1990] to show that the elliptic submanifolds (for the linearized vector field $L^{u}$ ) propagate the holomorphic extendability to wedges, as defined in [Trepreau, 1990], of the solution $u$.

\section{REFERENCES}

M. S. Baouendi, Ch. Chang, and F. Treves, Microlocal hypo-analyticity and extension of CR functions, J. Differential Geometry 18 (1983), 331-391.

M. S. Baouendi and F. Treves, A property of the functions and distributions annihilated by a locally integrable system of complex vector fields, Ann. of Math. (2) 113 (1981), 387-421.

J. Y. Chemin, Calcul paradifférentiel précisé et applications à des équations aux dérivées partielles non semilinéaires, Duke Math. J. 56 (1988), 431-469. 
N. Hanges and F. Treves, Propagation of holomorphic extendability of CR functions, Math. Ann. 263 (1983), 157-177.

J. M. Trepreau, Sur la propagation des singularités dans les variétés $C R$ preprint, 1990.

F. Treves, Hypo-analytic structures, Princeton Univ. Press, Princeton, N.J., 1992.

Department of Mathematics, City University of New York, Herbert H. Lehman ColLEGE, BRONX, NEW YORK 10468

Department of Mathematics, Rutgers University, New Brunswick, New Jersey 08903 